\title{
Mapeo científico en la búsqueda de información. Caso de estudio: enfermedades infecciosas en bovinos
}

\author{
Scientific Mapping in the Search for Information. Case Study: Infectious Diseases in \\ Bovines
}

\section{Mapeamento científico na busca de informações. Estudo de caso: doenças infecciosas em bovinos}

\author{
Javier-Antonio Ballesteros-Ricaurte \\ Eduardo Avendaño-Fernández ${ }^{2}$ \\ Angela-María González-Amarillo ${ }^{3}$ (C) \\ Adriana Granados-Comba ${ }^{4}$ (D)
}

Recibido: febrero de 2021

Aceptado: julio de 2021

Para citar este artículo: Ballesteros-Ricaurte, J. A., Avendaño-Fernández, E., González-Amarillo, A. M. y GranadosComba, A. (2021). Mapeo científico en la búsqueda de información. Caso de estudio: enfermedades infecciosas en bovinos. Revista Científica, 42(3), 265-275. https://doi.org/10.14483/23448350.17532

\section{Resumen}

Se plantean los pasos a seguir para realizar un mapeo científico sobre un tema de investigación utilizando herramientas que permitan identificar diferentes relaciones y análisis entre artículos, tomando como caso de estudio el tema de enfermedades infecciosas bovinas. La metodología utilizada se basa en seis pasos que van desde la recuperación de información por medio de la revisión sistemática de literatura hasta la visualización de los resultados por medio de la herramienta de análisis de mapas científicos SciMAT, empleando principalmente las palabras clave, el índice $\mathrm{H}$ y la ocurrencia de los términos en el texto. Se encuentra como resultado que los estudios sobre enfermedades infecciosas bovinas se han centrado en subtemas como brotes de la enfermedad, tratamientos utilizados, modelos de prevención y análisis de muestras de laboratorio. Por otro lado, se denota que hace falta realizar estudios sobre el uso de herramientas informáticas o el desarroIlo de aplicaciones que utilicen técnicas modernas como inteligencia artificial y machine learning como apoyo al análisis, el control y la erradicación de enfermedades infecciosas bovinas.

Palabras clave: análisis de co-palabras; enfermedades infecciosas; indicadores bibliométricos; índice h; SciMAT.

1. Ph. D. (c) Universidad Pedagógica y Tecnológica de Colombia, Tunja, Colombia, javier.ballesteros@uptc.edu.co.

2. Ph. D. Universidad Pedagógica y Tecnológica de Colombia, Tunja, Colombia, eduardo.avendano@uptc.edu.co.

3. M. Sc. Universidad Nacional Abierta y a Distancia - UNAD, Tunja, Colombia, angela.gonzalez@unad.edu.co.

4. M. Sc. Universidad Pedagógica y Tecnológica de Colombia, Tunja, Colombia, adriana.granados@uptc.edu.co. 


\section{Abstract}

The steps to be followed in a scientific mapping process on a research topic are proposed using tools that allow identifying different relationships and performing analyses between articles, taking the topic of infectious bovine diseases as a case study. The employed methodology is based on six steps that go from information retrieval through systematic literature review to the visualization of the results by means of the SciMAT scientific map analysis tool, mainly using the keywords, the $\mathrm{H}$ index, and the occurrence of the terms in the text. As a result, it is found that studies on infectious bovine diseases have focused on subtopics such as disease outbreaks, the treatments used, prevention models, and analysis of laboratory samples. On the other hand, it is indicated that further studies are needed on the use of computer tools or the development of applications that use modern techniques such as artificial intelligence and machine learning to support the analysis, control, and eradication of infectious bovine diseases.

Keywords: bibliometric indicators; co-word analysis; h-index; infectious diseases; SciMAT.

\section{Resumo}

Os passos a seguir para a realização de um mapeamento científico sobre um tema de pesquisa são propostos por meio de ferramentas que permitem identificar diferentes relações e análises entre artigos, tomando como estudo de caso o tema doenças infecciosas dos bovinos. A metodologia utilizada é baseada em seis etapas que vão desde a recuperação da informação por meio de uma revisão sistemática da literatura até a visualização dos resultados por meio da ferramenta de análise de mapas científicos SciMAT, utilizando principalmente as palavras-chave, o índice $\mathrm{H}$ e a ocorrência dos termos no texto. Como resultado, verifica-se que os estudos sobre doenças infecciosas bovinas têm enfocado subtemas como surtos de doenças, tratamentos utilizados, modelos de prevenção e análise de amostras laboratoriais. Por outro lado, nota-se que é necessária a realização de estudos sobre a utilização de ferramentas informáticas ou o desenvolvimento de aplicações que utilizem técnicas modernas como inteligência artificial e aprendizagem de máquina para apoiar a análise, controlo e erradicação de doenças infecciosas bovinas.

Palavras-chaves: análise de co-palavras; doenças infecciosas; indicadores bibliométricos; índice h; SciMAT.

\section{Introducción}

Los avances académicos y científicos se pueden observar en las revistas indexadas que son publicadas por las diferentes editoriales académicas; adicionalmente, las revistas son medios de comunicación en diferentes campos de la ciencia (Emami et al., 2018). En algunos casos la información que se encuentra en las bases de datos bibliográficas que reúnen los artículos de las revistas científicas es bastante y se hace necesario realizar un análisis de mapas científicos que permita conocer los avances en los diferentes campos del conocimiento, entregue información para la toma de decisiones y la formulación de nuevos proyectos de investigación (Montero-Díaz et al., 2018). En el sector lácteo se encuentran varios temas relevantes como diagnóstico, brotes, control y tratamientos de enfermedades, comportamiento de vacunas, áreas del conocimiento relacionadas con enfermedades, entre otros. Para el caso de estudio se toma las enfermedades infecciosas bovinas y las relaciones que se encuentran con otros campos del conocimiento.

Las enfermedades infecciosas bovinas generan problemas de salud pública y pérdidas económicas a los ganaderos (Ballesteros-Ricaurte et al., 2018; Machado et al., 2016), las asociaciones productoras de lácteos y, en general, a las personas y empresas que están vinculadas al sector lácteo (Ansari-Lari et al., 2017). Los temas relacionados con enfermedades infecciosas bovinas están enlazados con brotes, tratamientos y análisis de la presencia de la enfermedad en fincas (Al-Mamun et al., 2018)

Para conocer más de los diferentes temas y las relaciones que se presentan es necesario contar con una revisión sistemática por palabras clave, número de artículos publicados, índice h y 
co-ocurrencia. Por esta razón se realiza una revisión sistemática de literatura, para analizar la información y encontrar los temas más publicados y los que pueden ser innovadores. A partir de la combinación de palabras se realiza la búsqueda de información, teniendo en cuenta título, resumen, palabras clave y conclusiones en los artículos de revistas de diferentes áreas como ciencias computacionales, medicina veterinaria e informática incluidas en la base de datos Scopus, dando como resultado una recuperación de 237 artículos. Con esta información se realiza el proceso de análisis para obtener mapas de conocimiento.

Se propone realizar un análisis de mapa científico que proporcione una descripción general de la evolución conceptual del tema enfermedades infecciosas bovinas. El objetivo de este trabajo es relacionar diferentes herramientas en el proceso de revisión sistemática de literatura sobre enfermedades infecciosas bovinas, para conocer la base de conocimiento sobre este tema y la relación con otros temas, y así obtener un punto de partida para explorar aplicaciones en estos campos. Los análisis bibliométricos se utilizan para medir un campo de estudio en particular, por medio del análisis de citas y co-citas y las relaciones entre los subcampos en la literatura y basados en palabras; son útiles en el uso de modelos en la vida real y están diseñados para proporcionar un desarrollo de campo más preciso (Altıntaş et al., 2019).

Cobo et al. (2012) realizaron el estudio de diversas herramientas informáticas que sirven para obtener mapas científicos, resaltando la herramienta SciMAT, porque utiliza diferentes algoritmos y filtros en el análisis de los artículos. La creación de mapas científicos tiene como objetivo describir disciplinas específicas, dominios científicos o campos de investigación. Para explicar el estudio se cuenta con el desarrollo de seis pasos (recuperación de la información, pre-procesamiento, normalización de la red, mapeo, análisis y visualización) y el uso de la herramienta SciMAT (Cobo et al., 2011; Alcaide-Muñoz et al., 2017).
Por su parte, Dueñas et al. (2012) construyen el cuerpo de información a partir del conjunto de palabras clave utilizadas para realizar búsquedas recurrentes y relacionadas con otros artículos. Se define el número mínimo de relaciones para organizar los clústeres y se obtiene el mapa de conocimiento. El uso de gráficas de búsqueda de estudios es una forma de mostrar los resultados obtenidos de la revisión de literatura (Carrizo y Ortiz, 2016). Otra forma es realizar un conjunto de datos sobre las palabras clave siguiendo los trabajos presentados en congresos o en un grupo de revistas (Aguilar et al., 2020).

En el mapeo científico se pueden obtener diferentes tipos de resultados, por ejemplo Emami et al. (2018) se centraron en buscar información sobre grupos científicos de Oriente Medio que trabajaran en diabetes. Los grupos temáticos basados en índices globales y locales mostraron que la prevalencia de la diabetes, la hiperglucemia y los resultados del embarazo, los factores de riesgo de la diabetes, las complicaciones de la diabetes y sus nuevos tratamientos, y la monitorización de la glucosa en la diabetes fueron los campos que se abordaron en los principales artículos de los grupos de investigación.

Otra forma de realizar revisión sistemática de literatura es seleccionar las revistas, la definición de criterios de inclusión y exclusión de estudios, y la definición de categorías para el análisis. En el trabajo de Bacca et al. (2014) el objetivo de la revisión sistemática de literatura es presentar el estado actual de la investigación en realidad aumentada en educación. El estudio considera categorías para analizar el estado actual y las tendencias como los usos de la realidad aumentada en entornos educativos, así como ventajas, limitaciones, efectividad; la disponibilidad de procesos de adaptación y personalización en aplicaciones educativas, así como el uso de realidad aumentada para abordar las necesidades especiales de los estudiantes en diversos contextos. El resultado del análisis permitió sugerir tendencias, desafíos, posibilidades, oportunidades para futuras investigaciones y una visión general hacia el futuro. 


\section{Metodología}

Para realizar un análisis de mapeo científico o de ciencia (Vallejo-Correa et al., 2021), técnica bibliométrica para extraer conocimiento a partir de la información científica (Alcaide-Muñoz et al., 2017), se deben seguir seis pasos como se observa en la figura 1 y utilizar software especializado, los pasos a seguir son: recuperación de datos, pre-procesamiento de datos, extracción de red, normalización de red, mapeo, análisis y visualización. Al final de este proceso, los autores deben interpretar y obtener conclusiones de los resultados. Se encuentran diferentes herramientas informáticas como: VosViewer, SciMAT, con fortalezas en cada caso.

La herramienta SciMAT se utiliza en este trabajo, ya que usa diferentes algoritmos y diversas técnicas para generar redes e incorporar módulos para realizar los pasos de carga de archivos hasta la visualización de los resultados, genera diferentes redes de mapas para ver relaciones entre palabras, implementa diversas técnicas de preprocesamiento que permitirá detectar elementos similares que deben ser unificados, dividir los datos en diferentes periodos de tiempo, filtrar los datos para realizar el análisis con los datos más significativos y filtrar las redes para obtener las relaciones entre las unidades de análisis más importantes (Cobo et al. 2012).

Este estudio permite mostrar la relación que hay entre los diferentes temas, realizando una revisión de literatura relacionada; teniendo en cuenta diferentes técnicas citadas por (Patria et al., 2019), se procede a trabajar con el análisis de palabras clave y su correlación; la herramienta SciMAT entrega información de la relevancia de algunos temas respecto a las palabras clave utilizadas por medio de un mapa de relaciones y facilita establecer un marco teórico sólido que respalde la propuesta de investigación.

La elaboración de mapas de ciencia utilizando SciMAT reúne las principales ventajas de las herramientas informáticas que están basadas en el índice h y el análisis de co-palabras (Montero-Díaz et al., 2018). Adicionalmente, este estudio se basa en cuatro fases que realizan un análisis de rendimiento para detectar y visualizar los campos de investigación y la evolución temática. A partir de las palabras clave extraídas de los documentos de cada período se construye una red basada en la co-ocurrencia; posteriormente, se aplica un algoritmo de clustering para identificar los temas de investigación existentes.

Los temas identificados se representan gráficamente mediante un diagrama estratégico y una red temática. Los principales temas del campo y su evolución se representan a través de cambios en cada período. Como resultado se obtiene cada tema y área temática que pueden asociarse a un conjunto de documentos publicados, citas o diferentes tipos de índice $h$.

\section{Resultados}

La utilización de herramientas informáticas en diferentes áreas del conocimiento ha atraído el interés de investigadores y la introducción de tecnologías de la información y la comunicación (TIC) en diferentes sectores de interés del hombre y en especial en el sector salud (Wanden-Berghe et al., 2011), generando avances significativos, esto se puede evidenciar en diferentes estudios que se han publicado (Galvão et al., 2011).

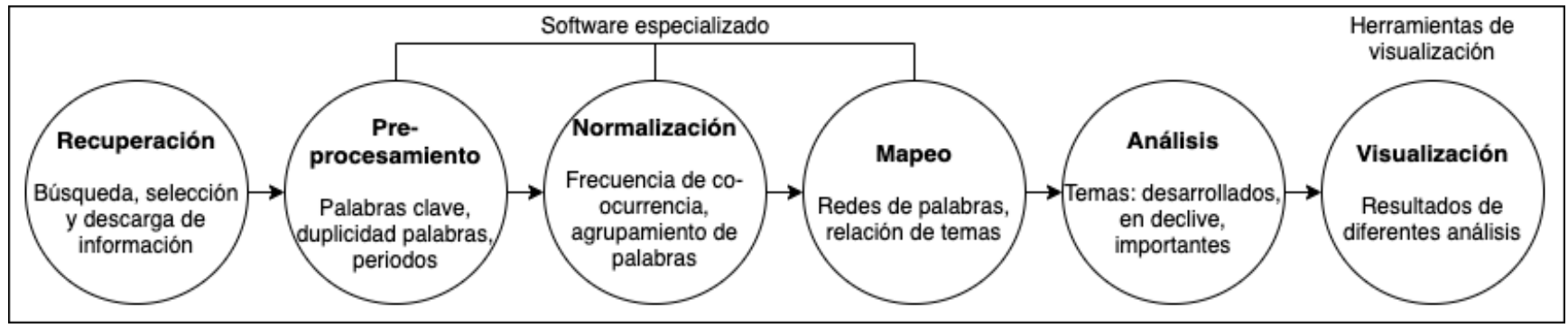

Figura 1. Pasos de la metodología 
La propuesta de este artículo es generar un estado del arte sobre el uso de modelos matemáticos en la epidemiología de enfermedades infecciosas en animales, teniendo en cuenta características como: tipo de modelo matemático, a qué animal está dirigido, tipo de enfermedad, modelo epidemiológico utilizado, entre otras. Para mostrar los resultados de este proceso, a continuación se desarrolla el uso de la metodología propuesta por Alcaide-Muñoz et al. (2017).

\section{Fase de recuperación de información}

Se revisaron publicaciones indexadas en Scopus en el periodo 2010-2017, utilizando la combinación "infectious disease" y "animals", con el formato de la búsqueda en título, palabras clave y resumen. Esta búsqueda generó un total de 13.627 artículos; para realizar el análisis hay que descargar el archivo plano que contiene la información de autores, año de publicación, datos de la revista, título del artículo, palabras clave y resumen. Scopus permite descargar archivos en diferentes formatos, pero tiene un límite en el tamaño del archivo, por esa razón se descargó un archivo por año de consulta. Para trabajar con la herramienta SciMAT se recomienda trabajar con archivos en formato RIS (Cobo et al., 2012)

\section{Fase de pre-procesamiento}

Los datos fueron pre-procesados teniendo en cuenta el formato de la herramienta SciMAT. Al cargar los archivos en la herramienta se pueden verificar las palabras clave, la duplicidad de palabras, la creación de grupos de palabras, no tener en cuenta palabras que están mal escritas, crear periodos de tiempo, por tanto y teniendo en cuenta que la información se descargó por años, se crearon cuatro periodos: 2010-2011, 2012-2013, 2014-2015 y 2016-2017. Por consiguiente, se realiza el proceso de reducción de datos para seleccionar la información más importante (en este caso se toman las palabras clave que más se acercan a la investigación) y se observa que algunas relaciones no tienen un suficiente significado para el análisis, entonces se eliminan los enlaces con menor peso (Cobo et al., 2012).

\section{Fase de normalización de la red}

En SciMAT hay un módulo para realizar este proceso, en el cual se selecciona la frecuencia de co-ocurrencia de palabras clave, agrupamiento de palabras (algoritmo de clustering basado en centros simples) con el fin de encontrar los grupos de palabras clave que están fuertemente relacionados con el tema de interés; la herramienta se encarga de generar las diferentes redes a partir de dos aspectos: centralidad que mide el grado de interacción de una red con respecto a otras redes y densidad que mide la fuerza interna de una red (Alcaide-Muñoz et al., 2017; Cobo et al. (2012)), siendo el grado de cohesión interna el tema de la red. En la figura 2 se observa la continuidad de las palabras clave en los diferentes periodos.

La flecha que sale del círculo representa las palabras clave que no se incorporan en el siguiente periodo; la flecha que ingresa al círculo representa las palabras clave nuevas; la flecha que conecta un círculo con el otro representa las palabras clave compartidas y el número al interior del círculo es la cantidad de palabras clave que se tuvieron en cuenta en ese periodo para la búsqueda.

\section{Fase de mapeo}

Como resultado de los procesos anteriores se obtiene un conjunto de grupos de palabras clave y sus interconexiones. SciMAT crea varias redes que se pueden observar al final del proceso, para entender la relación hay que tener en cuenta que la primera red es la evolución de los temas según los periodos de tiempo que se organizaron (figura 3). Cada columna representa un periodo (2010$2011,2012-2013,2014-2015$ y 2016-2017), las líneas sólidas que unen dos grupos indican que comparten el mismo nombre y el grosor de la línea depende del nivel de inclusión de ambos temas. La línea discontinua significa que los temas 


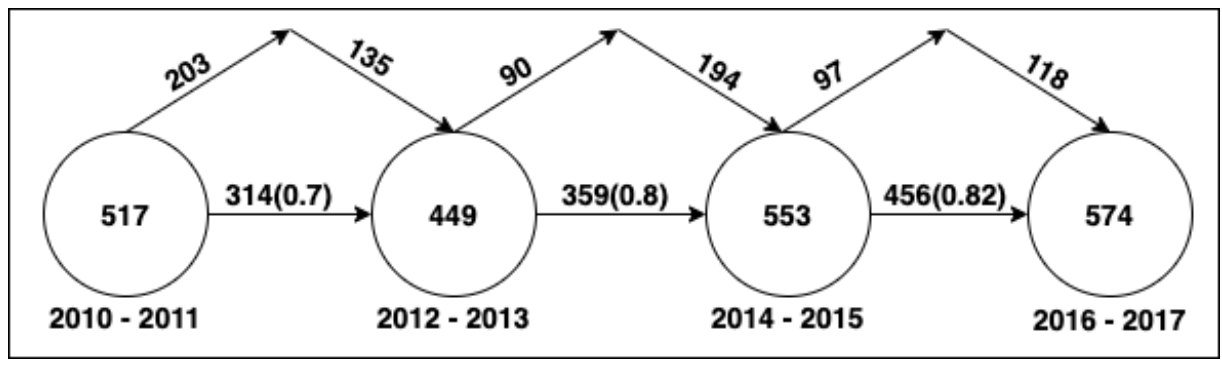

Figura 2. Continuidad de palabras clave entre periodos contiguos

comparten elementos que no son el tipo de temas (Cobo et al. 2012).

Después se visualizan redes por tema que se presentan en un plano, en la figura 4 se observan esferas que representan los temas y el tamaño de cada esfera depende del número de documentos relacionados; la ubicación en el plano depende de densidad y centralidad que se han aplicado, es decir, hay cuatro grupos: i) Grupo 1 (superior derecha), temas motores (en la figura 4 se muestran los temas "parásitos", "enfermedades infecciosas emergentes" como los que más trabajos relacionados tienen); ii) Grupo 2 (inferior derecha), temas básicos y transversales, entre los que "enfermedades infecciosas" con 31 trabajos representa el tema más importante; iii) Grupo 3 (inferior izquierda), temas emergentes o en desaparición ("vacunas" y "transmisión vertical" son los temas más representativos); y iv) Grupo 4 (superior izquierda), temas muy desarrollados y aislados ("respuesta inmune" es el tema que tiene más trabajos relacionados).

La figura 4 es un ejemplo de los diferentes diagramas que se pueden visualizar en la herramienta SciMAT, para observar cuál es la evolución de los temas en los diferentes periodos organizados y la relación con otros temas. Adicionalmente, estos diagramas sirven para identificar relaciones entre temas que están en ascenso y cuáles son de interés en la comunidad científica.

\section{Fase de análisis}

En varios estudios (Alcaide-Muñoz et al., 2017; Patria et al., 2019; Rincon-Patino et al., 2019) tienen en cuenta este tipo de análisis para identificar: temas desarrollados, temas en declive, temas transversales y temas de importancia para generar nuevas investigaciones, por ejemplo el periodo 2014-2015 que está representado en la figura 4 muestra que el tema "zoonosis" tiene fuerza y está generando investigaciones. Con este propósito, se utiliza el índice de inclusión para detectar nexos conceptuales entre temas de investigación en diferentes períodos y, de esta manera, identificar las áreas temáticas en un campo de investigación. Además, como cada tema está asociado con un conjunto de documentos, cada área temática también podría tener una colección asociada de documentos, obtenida mediante la combinación de documentos asociados con su conjunto de temas.

\section{Fase de visualización}

Siguiendo el flujo de trabajo del mapeo científico, se tomaron diferentes imágenes de la red mostrando relación de temas principales en la investigación y la relación con otros temas y los resultados de los diferentes análisis. Cuando se terminó el análisis de los mapas científicos, se analizaron los resultados y los mapas para agrupar los resultados y tomar los artículos más representativos en cuanto a los siguientes temas: modelo matemático, modelo epidemiológico, enfermedades infecciosas bovinas y las posibles combinaciones (figura 5), que son tema de estudio en el proyecto de investigación (Ballesteros-Ricaurte et al., 2018), para soportar explicar la relación de estos temas. 


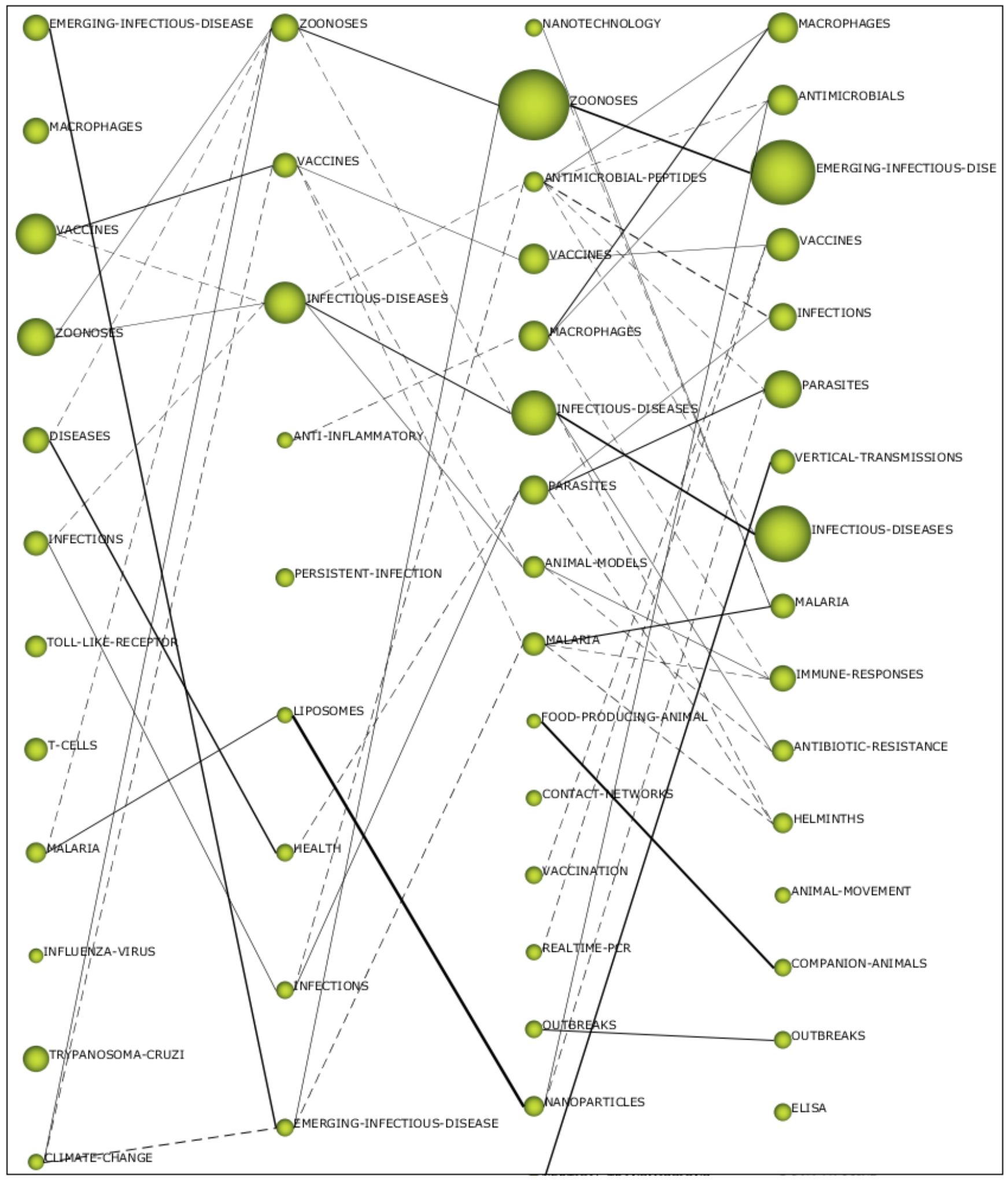

Figura 3. Evolución temática a partir de los campos de búsqueda "infectious disease" y "animals" en el periodo 2010-2017 


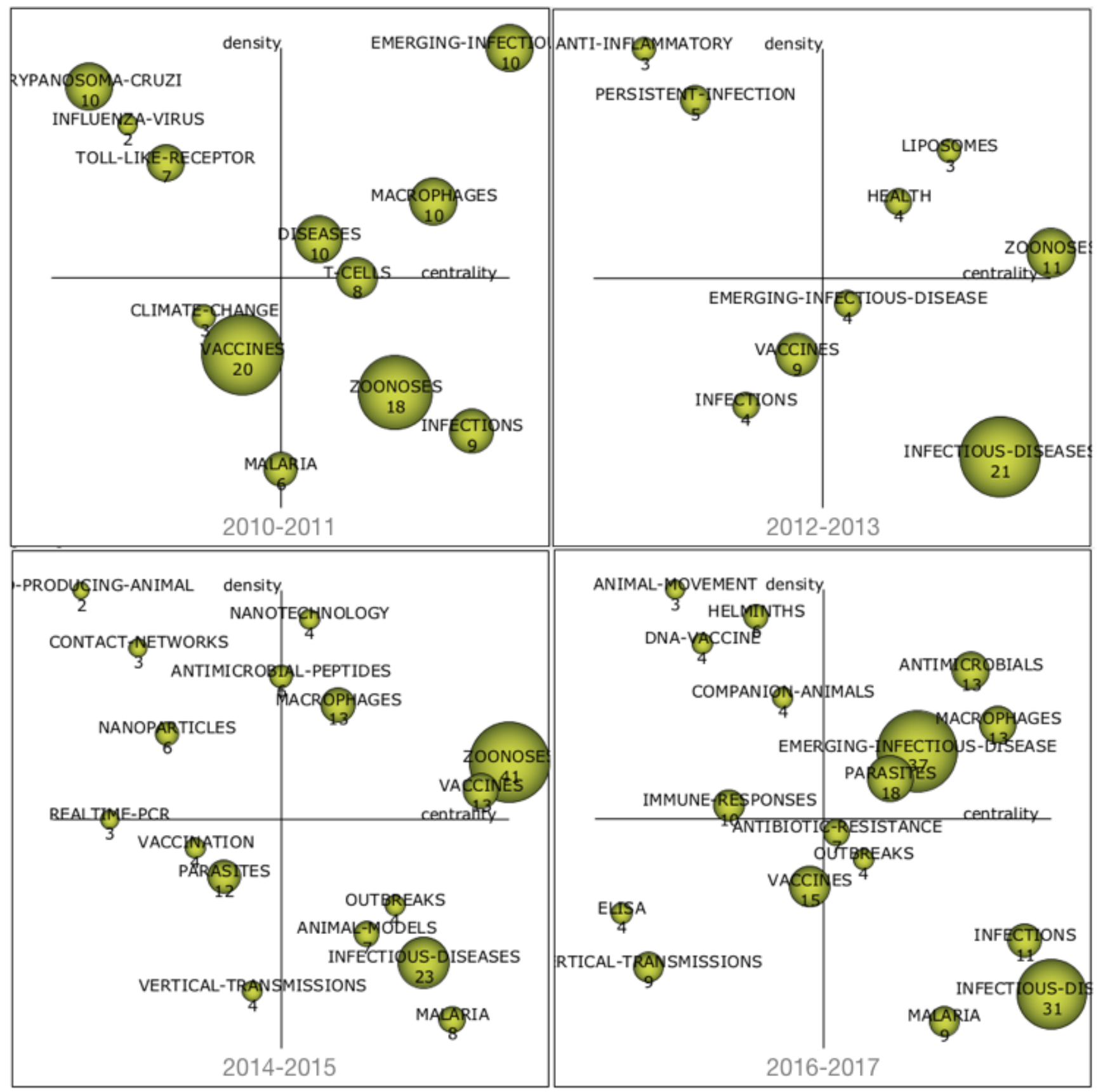

Figura 4. Diagrama estratégico de los periodos (2010-2011, 2012-2013, 2014-2015 y 2016-2017) basado en el número de documentos publicados, relacionados con el tema "infectious disease"

\section{Resultados del proceso de análisis}

El proceso sistemático de revisión de literatura se inició con la búsqueda en Scopus en la cual la combinación de "infectious disease" y "animals" en el título, palabras clave o resumen, arrojó 13.627 artículos. Esta información fue analizada con la ayuda de la herramienta SciMAT para encontrar relaciones entre las diferentes temáticas que tiene cada artículo y así seleccionar los artículos más relevantes sobre estos temas. Tomando las relaciones más relevantes entre las palabras clave y los temas 


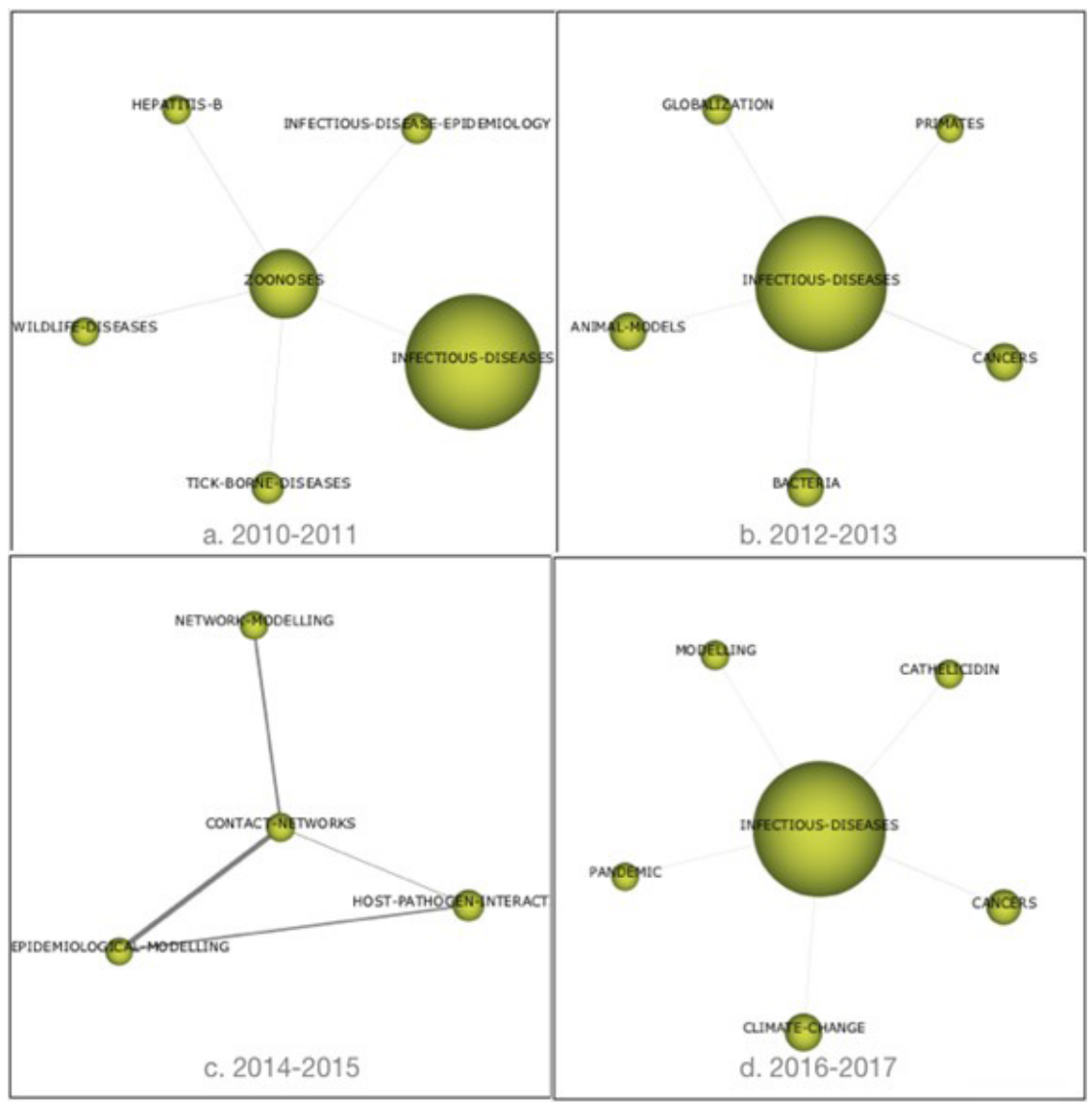

Figura 5. Diagrama de área temática por periodos. En cada diagrama se observa un tema principal y las relaciones con otros temas

Tabla 1. Resultado por período consultado

\begin{tabular}{|c|l|c|c|}
\hline Periodo & \multicolumn{1}{|c|}{ Temas principales encontrados } & Ecuación de búsqueda & \# artículos \\
\hline $2010-2011$ & zoonosis, infecciones, enfermedades humanas, epidemiología & & 38 \\
\cline { 1 - 2 } $2012-2013$ & enfermedades infecciosas, zoonosis, vacunas, modelos-animales & \multirow{2}{*}{$\begin{array}{c}\text { "infectious disease" }+ \\
\text { "models" + "bovines" }\end{array}$} & \multirow{2}{*}{638} \\
\hline $2014-2015$ & $\begin{array}{l}\text { zoonosis, enfermedades infecciosas, modelos-animales, } \\
\text { modelamiento de redes, epidemiología }\end{array}$ & & 70 \\
\hline $2016-2017$ & enfermedades infecciosas emergentes, vacunación, modelamiento & & 70 \\
\hline
\end{tabular}

que mostraron mayor importancia en el análisis, se siguió con una nueva búsqueda de información en Scopus considerando los periodos definidos y las relaciones de las palabras clave; en la tabla 1 se pueden observar los resultados de esta búsqueda.

Se le suma una búsqueda adicional para el año 2018, con la misma ecuación de búsqueda y se obtienen 28 artículos, para un total de 237 artículos. Pero las palabras clave que se utilizan en la búsqueda puede que no estén totalmente relacionadas con los temas principales de la investigación, por esta razón se aplica un nuevo filtro, en este caso manual (lectura del título, resumen y verificación de las palabras clave) para determinar cuáles de los artículos están directamente relacionados con los temas de investigación. El resultado 
de este filtro es un total de 67 artículos que están relacionados con modelos matemáticos y enfermedades infecciosas.

\section{Conclusiones}

En este trabajo solo se refleja la producción científica indexada en Scopus, en idioma inglés y una búsqueda centrada en enfermedades infecciosas, modelos y bovinos. No se tuvieron en cuenta sinónimos de las palabras clave o el tipo de revista en la que se encuentra publicado el artículo.

Las enfermedades infecciosas bovinas son un tema que se puede trabajar desde diferentes enfoques. En la literatura se encuentra información relacionada con tratamientos, brotes que se presentan en diferentes zonas geográficas, análisis de pruebas de sueros y algunos modelos epidemiológicos. A partir del análisis realizado se observa que hay varias relaciones con temas, por lo menos en número, que requieren ser estudiadas. También que hay temas nuevos que se pueden profundizar y son innovadores en este caso.

El mapeo científico permite visualizar las relaciones que se presentan a partir de las palabras clave seleccionadas y de nuevas palabras que aparecen, indicando el conocimiento relevante de los diferentes temas. Es un proceso que sirve para encontrar temas que han sido muy publicados y de posibles propuestas de trabajo de investigación. En trabajos futuros se debe realizar una comparación de estos resultados con muestras de otras bases de datos, teniendo en cuenta zonas geográficas y la selección de artículos en función de revistas.

\section{Referencias}

Aguilar, J., Salazar, C., Velasco, H., Monsalve-Pulido, J., Montoya, E. (2020). Comparison and Evaluation of Different Methods for the Feature Extraction from Educational Contents. Computation, 8(2), 1-20. https://doi.org/10.3390/computation8020030

Al-Mamun, M., Smith, R., Nigsch, A., Schukken, Y., Gröhn, Y. (2018). A Data-driven Individual-based
Model of Infectious Disease in Livestock Operation: A Validation Study for Paratuberculosis. PLOS ONE, 13(12), 1-18. https://doi.org/10.1371/journal. pone. 0203177

Alcaide-Muñoz, L., Rodríguez-Bolívar, M. P., Cobo, M. J., Herrera-Viedma, E. (2017). Analysing the Scientific Evolution of e-Government Using a Science Mapping Approach. Government Information Quarterly, 34(3), 545-555. https://doi.org/10.1016/j.giq.2017.05.002

Altıntaş, M. H., Kılıç, S., Akhan, C. E. (2019). The Transformation of the e-Tailing Field: A Bibliometric Analysis. International Journal of Retail \& Distribution Management, 48(2), 152-168. https://doi. org/10.1108/IJRDM-10-2018-0232

Ansari-Lari, M., Mohebbi-Fani, M., Lyons, N., Azizi, N. (2017). Impact of FMD Outbreak on Milk Production and Heifers' Growth on a Dairy Herd in Southern Iran. Preventive Veterinary Medicine, 144, 117-122. https://doi.org/10.1016/j. prevetmed.2017.05.022

Bacca, J., Baldiris, S., Fabregat, R., Graf, S., Kinshuk. (2014). Augmented Reality Trends in Education: A Systematic Review of Research and Applications. Educational Technology \& Society, 17(4), 133-149

Ballesteros-Ricaurte, J., Carrillo-Ramos, A., Parra, C., Gómez, J. (2018). Adaptive System to Support Decision-making of Dairy Ecosystem in Boyacá Department. Proceedings of the 14th International Conference on Web Information Systems and Technologies, 253-260. https://doi. org/10.5220/0006947502530260

Carrizo, D., Ortiz, C. (2016). Modelos del proceso de educción de requisitos: un mapeo sistemático. Ingeniería y Desarollo, 34(1), 184-203

Cobo, M., López-Herrare, A., Herrera-Viedma, E., \& Herrera, F. (2012). SciMAT: A new Science Mapping Analysis Software Tool. Journal of the American Society for Information Science and Technology, 63(8), 1609-1630. https://doi.org/10.1002/asi.22688

Cobo, M. J., López-Herrera, A. G., Herrera-Viedma, E., Herrera, F. (2011). Science Mapping Software Tools: Review, Analysis, and Cooperative Study Among Tools. Journal of the American Society for 
Information Science and Technology, 62(7), 13821402. https://doi.org/10.1002/asi.21525

Dueñas, M., Rojas, D., Eugenia Morales, M. (2012). Propuesta Metodológica para realizar Mapas de Conocimiento. Editorial Rev.Fac.Cienc.Econ, 1, 77-90

Emami, Z., Hariri, N., Khamseh, M. E., Nooshinfard, F. (2018). Mapping Diabetes Research in Middle Eastern Countries During 2007-2013: A Scientometric Analysis. Medical Journal of the Islamic Republic of Iran, 32(1), 486-494. https://doi.org/10.14196/ mjiri.32.84

Galvão, M., Ricarte, I., Daura, A. (2011). Tecnologia e informação em saúde: modelo de ensino-aprendizagem transdisciplinar. Perspectivas em Ciência da Informação, 16(4), 73-94. https://doi.org/10.1590/ S1413-99362011000400007

Machado, G., Egocheaga, R., Hein, H., Miranda, I., Neto, W., Almeida, L., Canal, C., Stein, M., Corbellini, L. (2016). Bovine Viral Diarrhoea Virus (BVDV) in Dairy Cattle: A Matched Case-Control Study. Transboundary and Emerging Diseases, 63(1), e1-e13. https://doi.org/10.1111/tbed.12219

Montero-Díaz, J., Cobo, M., Gutiérrez-Salcedo, M., Segado-Boj, F., Herrera-Viedma, E. (2018). Mapeo científico de la categoría "comunicación" en WoS
(1980-2013). Comunicar: Revista Científica de Comunicación y Educación, XXVI(55), 81-91. https:// doi.org/10.3916/C55-2018-08

Patria, H., Wahyuni, S., Kusumastuti, R. D. (2019). Intellectual Structure and Scientific Evolution of Strategic Decision in the Field of Business and Management (1971 to 2018). Asian Journal of Business and Accounting, 12(2), 233-286. https://doi. org/10.22452/ajba.vol12no2.9

Rincon-Patino, J., Ramirez-Gonzalez, G., Corrales, J. C. (2019). Exploring Machine Learning: A Bibliometric General Approach Using SciMAT. F1000Research, 7(1210), 1-21. https://doi.org/10.12688/ F1000RESEARCH.15620.1

Vallejo-Correa, P., Monsalve-Pulido, J., Tabares-Betancur, M. (2021). A Systematic Mapping Review of Context-aware Analysis and Its Approach to Mobile Learning and Ubiquitous Learning Processes. Computer Science Review, 39, e100335. https://doi.org/10.1016/j.cosrev.2020.100335

Wanden-Berghe, C., Sabucedo, L., Martínez de Victoria, I. (2011). Investigación virtual en salud: las tecnologías de la información y la comunicación como factor revolucionador en el modo de hacer ciencia. Salud Colectiva, 7(1), 29-38 